\title{
Hydroxytyrosol contributes to cell proliferation and inhibits apoptosis in pulsed electromagnetic fields treated human umbilical vein endothelial cells in vitro
}

\author{
YONG CHENG $^{1}$, ZHIWEI QU $^{1}, \mathrm{XIMENG} \mathrm{FU}^{2}$, QI JIANG ${ }^{2}$ and JIANFENG FEI ${ }^{2}$ \\ Departments of ${ }^{1}$ Bone Surgery and ${ }^{2}$ Plastic Surgery, The First Hospital of Harbin City, \\ Harbin, Heilongjiang 150010, P.R. China
}

Received September 14, 2016; Accepted May 16, 2017

DOI: $10.3892 / \mathrm{mmr} .2017 .7701$

\begin{abstract}
A variety of pulsed electromagnetic fields (PEMFs) have been experimentally and clinically used in an effort to promote wound healing, although the mechanisms involved remain unknown. The aim of the present study was to investigate the action of a novel protocol of co-treatment with PEMFs and hydroxytyrosol (HTY) on the proliferation and differentiation potential of human umbilical vein endothelial cells (HUVECs). The HUVECs were assigned randomly into three groups: Control, PEMF-treated and PEMF + HT-treated. The intensity of the electromagnetic field used in this protocol was $2.25 \mathrm{mT}$, the frequency of the bursts was $50 \mathrm{~Hz}$ and the application time was $15 \mathrm{~min}$. A Cell Counting kit-8 (CCK-8) assay was used to assess cell proliferation, and cell apoptosis was analyzed by TUNEL apoptosis assay kit and calcein-acetoxymethyl/propidium iodide dual-staining assay. In addition, protein and mRNA expression levels of protein kinase B (Akt), mechanistic target of rapamycin (mTOR), transforming growth factor (TGF)- $\beta 1$ and p53 were determined by western blotting and reverse transcription-quantitative polymerase chain reaction assays, respectively. The CCK-8 assay demonstrated that HTY contributed to HUVEC proliferation mediated by PEMFs in a time-dependent manner. The Transwell assay and scratch wound results demonstrated that co-treatment of HTY and PEMFs could increase HUVEC migration. Furthermore, the levels of apoptotic cells were reversed by pre-incubation with HTY in the PEMF treatment group, while PEMF treatment alone had no such effect. The proteins and mRNA expression levels of Akt, mTOR, TGF- $\beta 1$ were elevated in co-treatment of HTY and PEMFs, whereas there was no effect on levels of p53. Therefore, the results indicated that combined exposure
\end{abstract}

Correspondence to: Dr Jianfeng Fei, Department of Plastic Surgery, The First Hospital of Harbin City, 151 Diduan Street, Daoli, Harbin, Heilongjiang 150010, P.R. China

E-mail: chengyong7890@163.com

Key words: pulsed electromagnetic fields, human umbilical vein endothelial cells, hydroxytyrosol, proliferation, migration, apoptosis of HUVECs to PEMFs and HTY exerted protective effects in HUVECs by promoting cell proliferation and inhibiting apoptosis. In conclusion, to the best of our knowledge, the present study was the first to demonstrate the beneficial roles of HTY and PEMF combined treatment in HUVECs, which may represent an effective treatment for wound healing.

\section{Introduction}

Wound healing is clinically defined as the completion of the closure of the wounded skin area, which is an interactive and dynamic process. It includes phases of hemostasis, cellular proliferation, inflammation, angiogenesis, matrix synthesis and remodeling, and formation of granulation tissue (1). The evolution of the wound healing process involves a series of phenomena which represent attempts in reestablishing the anatomical structure and function of the normal tissue. One aspect of wound repair that has always been considered to be essential for adequate healing is the creation of new vasculature via angiogenesis (2).

Understanding the correct sequence of molecular and cellular processes that occur during wound healing is critical for the development of novel treatments. Previous studies have demonstrated that pulsed electromagnetic fields (PEMFs) are capable of altering the structure of the cell membranes, and diversifying the permeability of different ion channels and the potential of the cellular membranes. At the molecular and cellular level, PEMFs have been advocated to exert a direct effect on the production of proteins and promote the synthesis of extracellular matrix proteins that regulate gene transcription (3). Additionally, previous studies have suggested that the process of angiogenesis, both in vivo and in vitro, may be influenced by various forms of electrical stimulation, including direct current, combined electromagnetic fields and PEMFs (4). In another study, Athanasiou et al (3) demonstrated that short duration PEMFs appear to facilitate and improve the quality of skin wound healing in a rat model. Nevertheless, further studies are required to define the optimal characteristics of the PEMFs, in order to ensure a faster and more effective wound healing process. Furthermore, PEMFs may also affect several membrane receptors and stimulate endothelial cells to secrete several growth factors, including vascular endothelial growth 
factor, connective tissue growth factor and endothelial nitric oxide synthase in vivo and in vitro $(5,6)$. In vitro, $\mathrm{PEMFs}$ have been demonstrated to inhibit the process of hypoxia-induced apoptosis and augmented tube formation, migration and proliferative capacities of human umbilical vein endothelial cells (HUVECs) (7). To date, PEMFs have been applied experimentally and clinically to promote wound healing for many years $(8,9)$.

Hydroxytyrosol (HTY), or 3,4-dihydroxyphenylethanol, is one of the hydroxyaromatic components of secoiridoids, which is a very bioactive alcoholic ortho-diphenol. Accumulating experimental, clinical and epidemiological data have indicated that HTY is antioxidant and antimicrobial and that it has beneficial effects on the cardiovascular system and in several human diseases $(10,11)$. Zrelli et al (12) revealed that HTY upregulates heme oxygenase- 1 expression by stimulating the nuclear accumulation and stabilization of nuclear factor erythroid 2-related factor 2, which leads to the wound repair of vascular endothelial cells crucial in the prevention of atherosclerosis. In another study, Scoditti et al (13) revealed that HTY could reduce inflammatory angiogenesis in cultured endothelial cells, through matrix metalloproteinase- 9 and cyclooxygenase-2 inhibition, supporting a potential protective role for dietary polyphenols in atherosclerotic vascular disease and cancer. However, the exact function of HTY on endothelial cells has not been fully uncovered until now, especially in association with co-treatment with PEMFs.

When developing a wounding and healing model for PEMFs studies, endothelial cells appear to be an ideal system to investigate (14). The present study investigated the impact of the co-treatment of HYT and PEMFs on HUVEC function, and evaluated their influence on proliferation and migration in primary cultures of HUVECs. The present study also investigated the expression of functional parameters such as migration, viability and apoptosis, and the gene expression of protein kinase $\mathrm{B}$ (Akt), mechanistic target of rapamycin (mTOR), transforming growth factor (TGF)- $\beta 1$ and p53 under the influence of different doses of HYT and PEMFs on HUVECs. The results demonstrated that PEMFs may provide novel research options in the field of wounding healing by promoting endothelial cell growth and by enhancing the healing response of the endothelium.

\section{Materials and methods}

Cell culture. HUVECs were obtained from the American Type Culture Collection (Manassas, VA, USA). The HUVECs were cultured in Dulbecco's modified Eagle's medium (DMEM) supplemented with $10 \%$ fetal bovine serum (FBS; HyClone; GE Healthcare Life Sciences, Logan, UT, USA). The cells were cultured in $95 \%$ air and $5 \%$ carbon dioxide at $37^{\circ} \mathrm{C}$.

PEMF exposure. The PEMFs used in the current study was one that was clinically available for the treatment of wound healing. The devices were supplied by Electro-Biology, Inc. (Parsippany, NJ, USA). The model required a voltage of $230 \mathrm{~V}$ with a frequency of $50 / 60 \mathrm{~Hz}$ to generate PEMF with a maximum intensity of $0.015 \mathrm{~T}$ (150 Gauss) and an impulse repetition frequency of up to $100 \mathrm{~Hz}$. At the beginning of the experiment, the intensity of the magnetic fields generated inside the solenoid was measured with a Gaussimeter PCE-G28 (PCE Group, Albacete, Spain) with a triaxial probe. The intensity of the electromagnetic field used in this protocol was $2.25 \mathrm{mT}$, the frequency of the bursts was $50 \mathrm{~Hz}$ and the application time was $15 \mathrm{~min}$. PEMF irradiation was performed on days 1,2,3 and 4 of culture, with the solenoid inside the incubator, under the same conditions of temperature.

Cell viability assay. The cell viability was calculated using the Cell Counting kit-8 (CCK-8; Beyotime Institute of Biotechnology, Jiangsu, China) according to the manufacturer's protocol. Briefly, HUVECs $\left(1 \times 10^{4} /\right.$ well) were seeded into 96-well plates in DMEM supplemented with $10 \%$ FBS for different time points. Following this, the cells were exposed to PEMFs at days $0,1,2,3$ or 4 , or treated with $\operatorname{HTY}(0,10$, $30,50,100,150 \mu \mathrm{M})$ at day 2 , or treated with a combination on days $0,1,2$ or 4 . Finally, the viable cells were detected using by CCK- 8 assay, where the absorbance for each sample was assessed at $450 \mathrm{~nm}$ using a microplate reader (Tecan, Männedorf, Switzerland).

Cell migration analysis. For the migration assay, cells were treated with PEMFs/HTY or a combination for $48 \mathrm{~h}$, and then re-suspended cells were removed on the top layer of a Transwell chamber with $8 \mu \mathrm{m}$ pores (EMD Millipore, Billerica, MA, USA). The lower layer of the chamber contained $10 \% \mathrm{FBS}$ as a chemoattractant. The chambers were placed at $37^{\circ} \mathrm{C}$ in $5 \%$ $\mathrm{CO}_{2}$ for $48 \mathrm{~h}$. Non-migrating cells on the top of the membrane were removed with cotton swabs. Cells that had migrated to the bottom were fixed with $95 \%$ ethanol, stained with $0.2 \%$ crystal violet staining (Sigma-Aldrich; Merck KGaA, Darmstadt, Germany), and imaged by bright-field microscopy (IX71; Olympus Corporation, Tokyo, Japan) and counted using Image-Pro Plus v6.0 software (Media Cybernetics, Inc., Rockville, MD, USA). Each experiment was performed in triplicate.

TUNEL assay. DNA fragmentation in HUVECs was detected by TUNEL assay using a Cell Death Detection kit (Roche Applied Science, Mannheim, Germany). Briefly, air-dried slides were fixed with $4 \%$ paraformaldehyde for $30 \mathrm{~min}$ at room temperature and cleaned three times with PBS for $10 \mathrm{~min}$, and then permeabilized with $1 \%$ Triton $\mathrm{X}-100$ for $4 \mathrm{~min}$ at $4^{\circ} \mathrm{C}$. Then, the TdT-labelled nucleotide mix was added to each slide and incubated at $37^{\circ} \mathrm{C}$ for $60 \mathrm{~min}$ in dark. Slides were washed twice with PBS and then counterstained with $10 \mathrm{mg} / \mathrm{ml} \mathrm{4,6-diamidino-2-phenylindole} \mathrm{for} 5 \mathrm{~min}$ at $37^{\circ} \mathrm{C}$.

Calcein-acetoxymethyl (AM)/propidium iodide (PI) dual-staining assay. A calcein-AM/PI dual-staining assay (Invitrogen; Thermo Fisher Scientific, Inc., Waltham, MA, USA) was performed to verify the effect of PEMFs and HTY on cell apoptosis. The calcein-AM/PI/CoCl${ }_{2}$ assay relies on the intracellular esterase activity within living cells. Living cells are stained with green fluorescence, and dead cells are stained red by PI. HUVECs were treated with PEMFs and HTY for $48 \mathrm{~h}$ in DMEM with $10 \%$ FBS. Fluorescence was analyzed by microscopy (Olympus Corporation). 

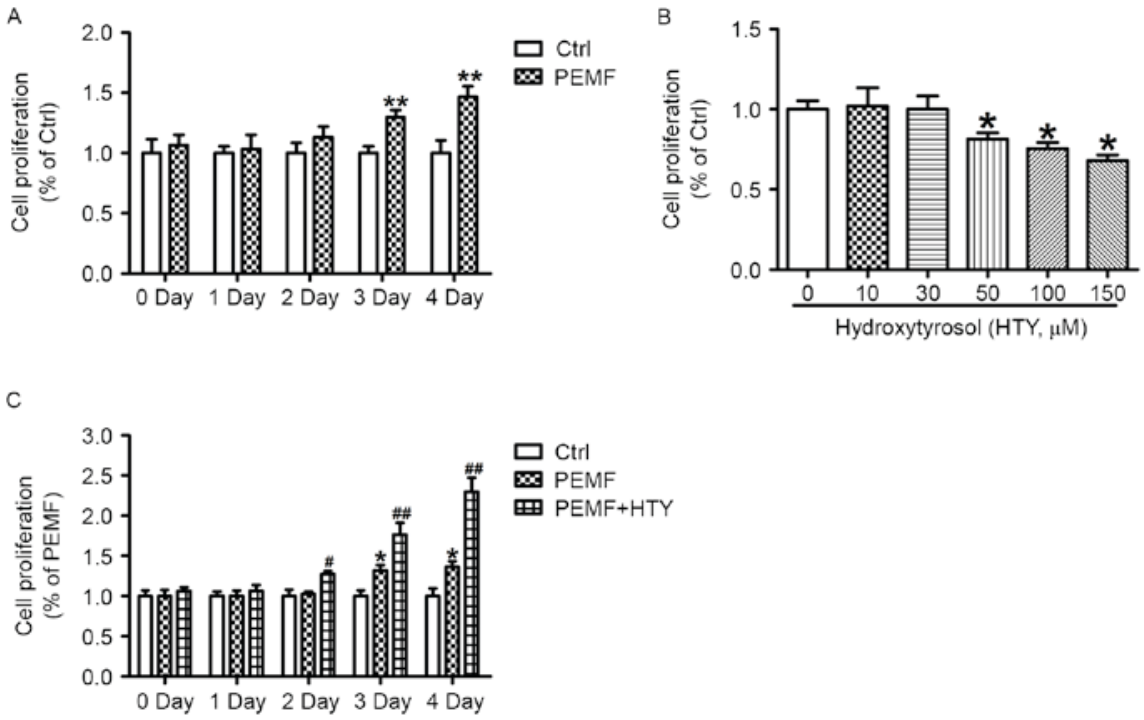

Figure 1. Combined HTY and PEMF treatment promotes cell proliferation in human umbilical vein endothelial cells. Cell proliferation following: (A) PEMF treatment across various time points ( ${ }^{* *} \mathrm{P}<0.01$ vs. Ctrl); (B) HTY treatment at various concentrations [ $\mathrm{P}<0.05$ vs. HTY $(0 \mu \mathrm{M})$ treatment group]; and (C) combined HTY and PEMF treatment ( $\mathrm{P}<0.05$ vs. Ctrl; ${ }^{*} \mathrm{P}<0.05,{ }^{\# /} \mathrm{P}<0.01$ vs. PEMF-treated group). Data are expressed as the mean \pm standard error of six independent experiments. HTY, hydroxytyrosol; PEMFs, pulse electromagnetic fields; Ctrl, control.

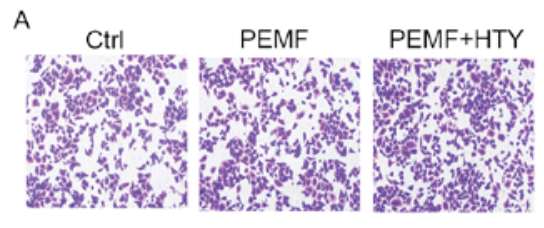

B
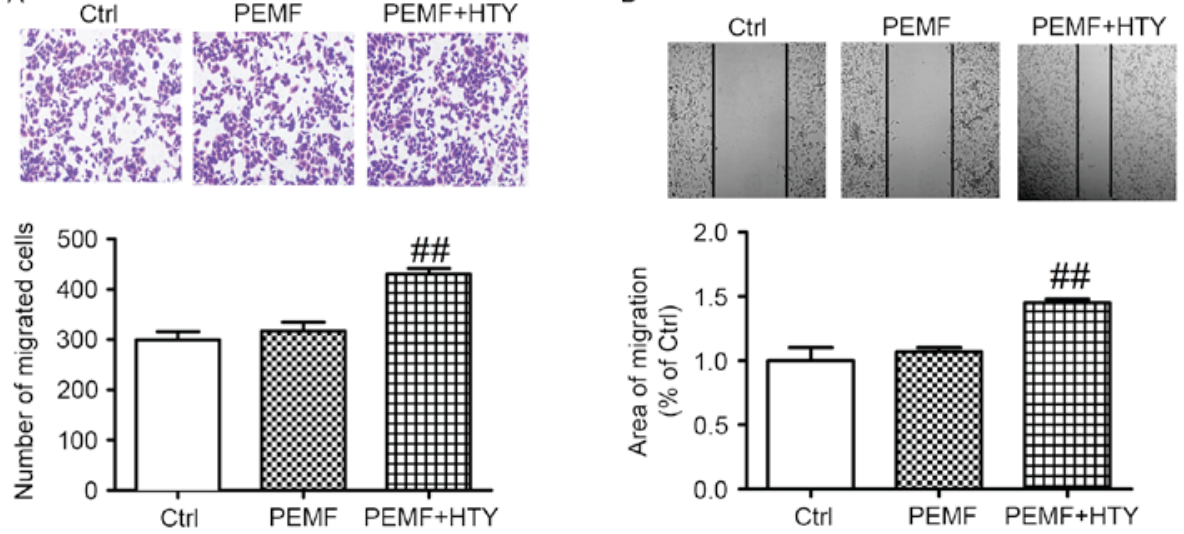

Figure 2. Effect of HTY and PEMF on cell migration in human umbilical vein endothelial cells. Representative images (magnification, x200) and quantification from (A) Transwell migration and (B) scratch wound assays. Data are expressed as the mean \pm standard error of six independent experiments. ${ }^{\# \#} \mathrm{P}<0.01 \mathrm{vs}$. PEMF-treated group. HTY, hydroxytyrosol; PEMFs, pulse electromagnetic fields; Ctrl, control.

Reverse transcription quantitative-polymerase chain reaction (RT-qPCR). Total RNA samples from cultured HUVECs were isolated using TRIzol reagent (Invitrogen; Thermo Fisher Scientific, Inc.), according to the manufacturer's protocols. Total RNA $(1 \mu \mathrm{g})$ was then reverse transcribed using the High-Capacity cDNA Reverse Transcription kit (Toyobo Co., Ltd., Osaka, Japan) to obtain cDNA, with the following temperature protocol: $37^{\circ} \mathrm{C}$ for $15 \mathrm{~min}$, then $98^{\circ} \mathrm{C}$ for $5 \mathrm{~min}$. The SYBR Green PCR Master Mix kit (Toyobo Co., Ltd.) was used for qPCR to quantify RNA levels of silent information regulator 1 (SIRT1). GAPDH served as an internal control. qPCR was performed on the 7300 FAST Real-Time PCR system (Applied Biosystems; Thermo Fisher Scientific, Inc.) for 40 cycles. The thermocycling conditions were as follows: $95^{\circ} \mathrm{C}$ for $10 \mathrm{~min}$ followed by $95^{\circ} \mathrm{C}$ for $15 \mathrm{sec}$, then 40 cycles of $60^{\circ} \mathrm{C}$ for $30 \mathrm{sec}$ and $72^{\circ} \mathrm{C}$ for $30 \mathrm{sec}$. The results were quantified using the $2^{-\Delta \Delta \mathrm{Cq}}$ method (15). Primer sequences were as follows: Akt, forward, 5'-GGTGATCCTGGTGAAGGAGA-3' and reverse, 5'-CTTAATGTGCCCGTCCTTGT-3'; mTOR, forward, 5'-TTCTGGTGCGACACCGAATC-3' and reverse 5'-CATCGGGTTGTAGGCCTGTG-3'; TGF- $\beta 1$, forward, 5'-CCCCGAGGGCGGCATG-3' and reverse, 5'-CATGCC GCCCTCGGGG-3'; p53, forward, 5'-ACGACGGTGACA CGCTTCCCTG-3' and reverse, 5'-CGCTAGGATCTGACT GCGGCTC-3'; and GAPDH, forward, 5'-GCTCTCTGCTCC TCCTGTTC-3' and reverse, 5'-ACGACCAAATCCGTTGAC TC-3'.

Scratch-wound assay. The confluent HUVECs cultured in 6-well plates were scratched with pipette tips, which led to a 1-mm-wide lane per well, and the ablate cells were cleaned with PBS. Subsequently, the cells were treated with or without hydroxytyrosol (Shanghai Pureone Biotechnology Co., Ltd, Shanghai, China) with DMEM supplemented with $10 \%$ 


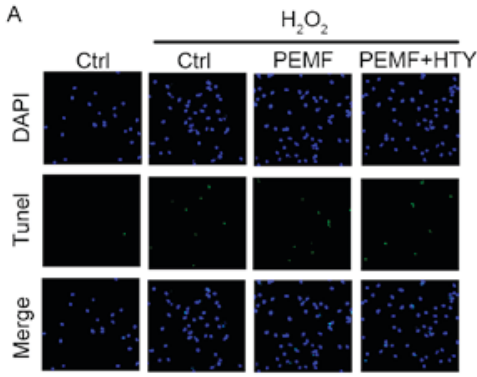

C

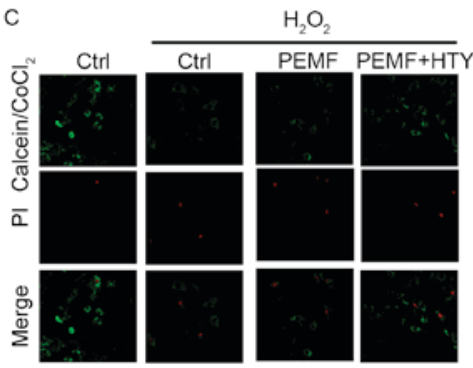

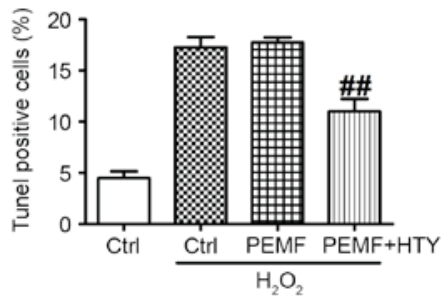

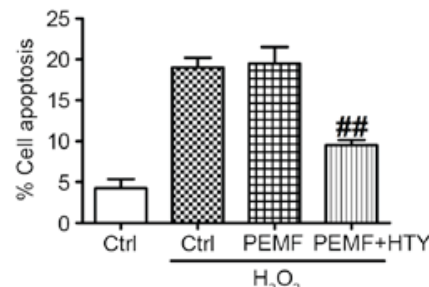

Figure 3. Effect of HTY and PEMF on cell apoptosis under $\mathrm{H}_{2} \mathrm{O}_{2}$ stimulation in human umbilical vein endothelial cells. (A) Representative fluorescence images and (B) quantification of TUNEL-positive cells. DAPI staining represents the nucleus. (C) Representative fluorescence images and (D) quantification of the ratio of apoptotic cells at $24 \mathrm{~h}$, as assessed by calcein acetoxymethyl/PI staining. Data are expressed as the mean \pm standard error of six independent experiments. ${ }^{\# \#<0.01 ~ v s . ~ P E M F-t r e a t e d ~ g r o u p . ~ M a g n i f i c a t i o n, ~} \mathrm{x} 200$. HTY, hydroxytyrosol; PEMFs, pulse electromagnetic fields; Ctrl, control; PI, propidium iodide; TUNEL, terminal deoxynucleotidyl transferase dUTP nick-end labeling.

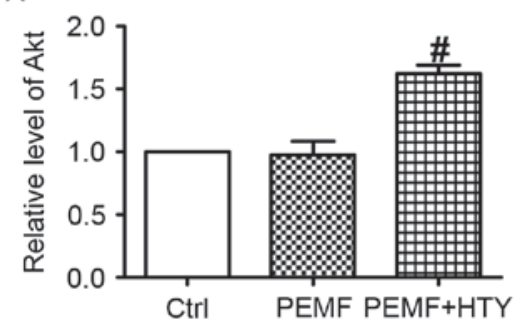

C

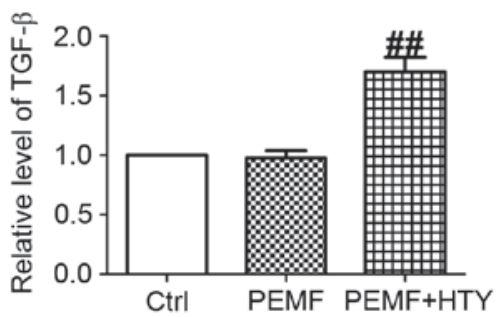

B

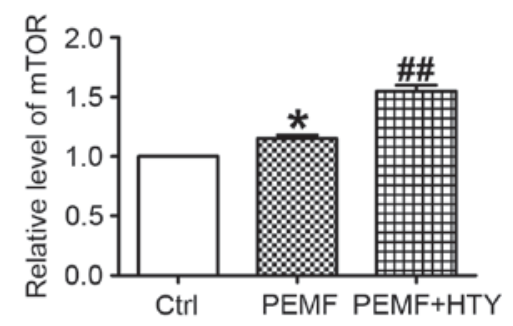

D

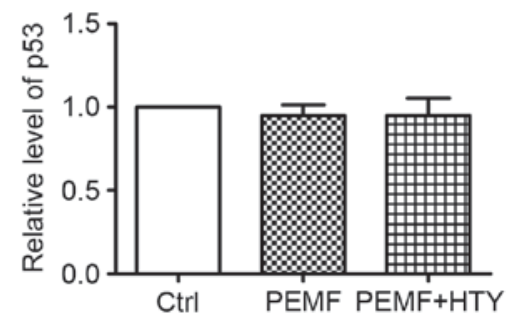

Figure 4. Effect of HTY and PEMF on mRNA expression levels of Akt, mTOR, TGF- $\beta$ and p53 at $24 \mathrm{~h}$. (A) Akt, (B) mTOR, (C) TGF- $\beta$ and (D) p53 mRNA expression levels in human umbilical vein endothelial cells. Data are expressed as the mean \pm standard error of six independent experiments. "P $<0.05$ vs. $\mathrm{Ctrl} ;{ }^{\# \mathrm{P}}<0.05,{ }^{\# \#} \mathrm{P}<0.01$ vs. PEMF-treated group. HTY, hydroxytyrosol; PEMFs, pulse electromagnetic fields; Ctrl, control; Akt, protein kinase B; mTOR, mechanistic target of rapamycin; TGF- $\beta$, transforming growth factor- $\beta$.

FBS at $37^{\circ} \mathrm{C}$ for $1-4$ days. Wounded areas were imaged by bright-field microscopy (magnification, x200; IX71 Olympus) and analyzed using Image-Pro Plus v6.0 software (Media Cybernetics, Inc.) at time point zero and after a $48 \mathrm{~h}$ treatment.

Western blot analysis. HUVECs were treated with PEMFs or HTY in DMEM with $10 \%$ FBS for 48 h. Proteins were solubilized and extracted with $200 \mu \mathrm{l}$ lysis buffer (Beyotime Institute of Biotechnology) and kept for 20 min on ice. Following this, the lysates were sonicated and centrifuged at $12,000 \mathrm{x} \mathrm{g}$ for $15 \mathrm{~min}$ at $4^{\circ} \mathrm{C}$, and the insoluble fractions were discarded. Equal amounts of protein $(\sim 60 \mu \mathrm{g})$ from each sample were separated by $10 \%$ SDS-PAGE, and transferred onto a nitrocellulose membrane (EMD Millipore). After a $1 \mathrm{~h}$ incubation with $5 \%$ non-fat dry milk powder, the membranes were probed with the following primary antibodies: anti-GADPH $(1: 5,000$ dilution; cat. no. 97166), anti-Akt (1:2,000; cat. no. 9272), anti-mTOR (1:1,000; cat. no. 2983), anti-human TGF- $\beta 1$ 


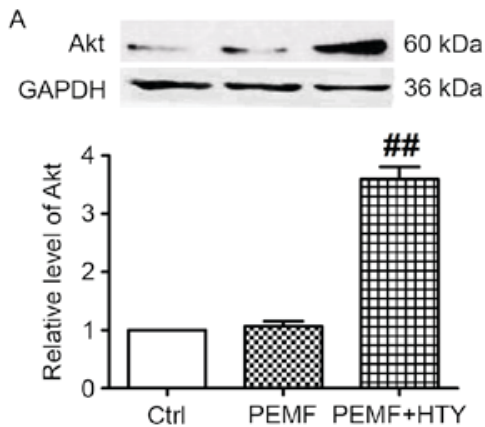

C
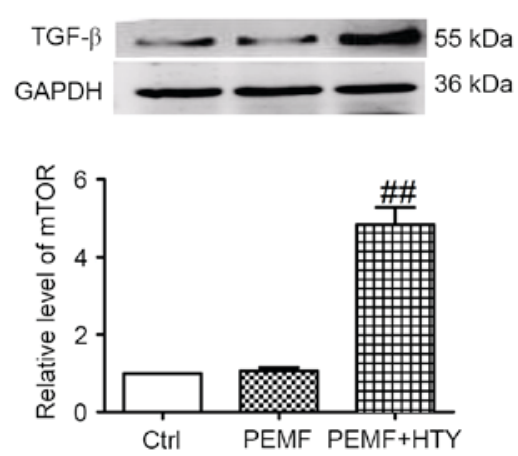

B
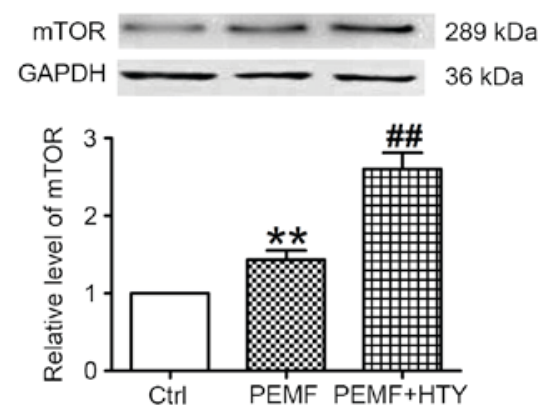

D
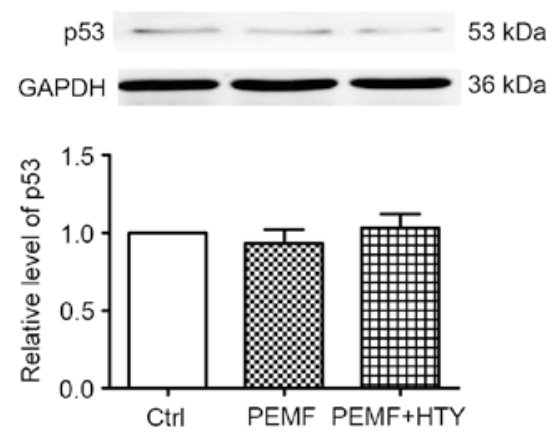

Figure 5. Effect of HTY and PEMF on protein expression levels of Akt, mTOR, TGF- $\beta$ and 553 at $24 \mathrm{~h}$. Representative western blot images and quantification of (A) Akt, (B) mTOR, (C) TGF- $\beta$ and (D) p53 protein expression levels in human umbilical vein endothelial cells. Data are expressed as the mean \pm standard error of three independent experiments. ${ }^{* *} \mathrm{P}<0.01$ vs. Ctrl; ${ }^{\# \#} \mathrm{P}<0.01$ vs. PEMF-treated group. HTY, hydroxytyrosol; PEMFs, pulse electromagnetic fields; Ctrl, control; Akt, protein kinase B; mTOR, mechanistic target of rapamycin; TGF- $\beta$, transforming growth factor- $\beta$.

(1:2,000; cat. no. 3711) and anti-p53 (1:2,000; cat. no. 2524; all Cell Signaling Technology, Inc., Danvers, MA, USA) overnight at $4^{\circ} \mathrm{C}$. Membranes were washed three times for $10 \mathrm{~min}$ in PBS containing $0.5 \%$ Tween-20, then incubated for $1 \mathrm{~h}$ at room temperature with the following Alexa Fluor ${ }^{\mathrm{TM}}$-conjugated secondary antibodies: IRDye ${ }^{\circledR} 800 \mathrm{CW}$ goat anti-rabbit $\mathrm{IgG}$ conjugated to IRDye ${ }^{\circledR} 800 \mathrm{CW}$ (cat. no. 926-32211; 1:10,000 dilution; LI-COR Biosciences, Lincoln, NE, USA) and IRDye ${ }^{\circledR}$ $800 \mathrm{CW}$ goat anti-mouse IgG conjugated to IRDye ${ }^{\circledR} 800 \mathrm{CW}$ (cat. no. 926-32210; 1:10,000 dilution; LI-COR Biosciences). The bands were visualized using the Odyssey Imaging system (LI-COR Biosciences) and semi-quantified using the Odyssey software version 3.0. Relative protein expression was normalized to that of GAPDH, which was used as an internal control, and experiments were repeated three times, unless otherwise stated.

Statistical analysis. All quantitative data are expressed as the mean \pm standard error. Statistical analysis was performed using SPSS version 19.0 (IBM Corp., Armonk, NY, USA). A Student's t-test or one-way analysis of variance followed by Dunnett's test was performed where appropriate. $\mathrm{P}<0.05$ was considered to indicate a statistically significant difference.

\section{Results}

HTY promotes HUVEC proliferation mediated by PEMFs in a time-dependent manner. As endothelial cell proliferation represents one of the critical processes for wound healing, the present study investigated the effect of PEMFs for $<24 \mathrm{~h}$ on the cell proliferation of HUVECs. PEMFs increased the proliferation rate of HUVECs in a time-dependent manner, significantly so after 72 and 96 h of exposure (Fig. 1A). In order to investigate whether HTY could promote proliferation induced by treatment with PEMFs, different dosages of HTY was used. The results demonstrated that 50, 100 and $150 \mu \mathrm{M}$ HTY could markedly reduce cell proliferation at the $24 \mathrm{~h}$ time point (Fig. 1B). Therefore, $30 \mu \mathrm{M}$ HTY was selected for following experiments. As presented in Fig. 1C, HTY could substantially increase the cell proliferation in PEMF-treated cells at the $24 \mathrm{~h}$ time point, while PEMF treatment alone produced no response on cell proliferation.

HTY enhances HUVEC migration induced by PEMF. To further determine the effect of HTY on cell migration, Transwell and scratch wound assays were performed. The results demonstrated that the number of migratory cells was significantly enhanced following combined HTY and PEMF treatment, compared with the control and PEMF treatment only (Fig. 2).

Co-treatment of HTY and PEMFs has anti-apoptosis effects, and ameliorates mitochondrial dysfunction induced by $\mathrm{H}_{2} \mathrm{O}_{2}$. An increase in apoptotic cells is associated with wound healing. Therefore, a TUNEL assay was performed. Briefly, HUVECs were treated with HTY/PEMFs or combined with $\mathrm{H} 2 \mathrm{O} 2$, (representative images in Fig. 3A). The results demonstrated that combined PEMF and HTY could significantly reduce the number of apoptotic HUVECs, while PEMF treatment alone produced no effects (Fig. 3B). In addition, calcein/PI/CoCl 2 staining was used to verify cell apoptosis associated with mitochondrial dysfunction (representative 
images are presented in Fig. 3C). In Fig. 3D, apoptotic cells were markedly decreased in the PEMF- and HTY-treated group, while PEMFs administered alone had no effect on cell apoptosis. Therefore, the anti-apoptotic effect of HTY may be associated with ameliorating mitochondrial dysfunction.

$H T Y$ increases the transcription activities of Akt, mTOR and $T G F-\beta$, but not $p 53$, in HUVECs. To clarify the anti-apoptosis and pro-proliferation effects of HTY following PEMF treatment, the mRNA expression levels of Akt, mTOR, TGF- $\beta$ and p53 were detected. Combined PEMF and HTY treatment significantly increased the mRNA expression levels of Akt (Fig. 4A), mTOR (Fig. 4B) and TGF- $\beta$ (Fig. 4C) compared with the control. In addition, the mRNA expression of mTOR was substantially elevated following both HTY and PEMF treatment (Fig. 4B). Notably, no significant differences were observed in the mRNA expression levels of p53 (Fig. 4D).

HTY increases the protein expression levels of Akt, mTOR and TGF- $\beta$, but not 553 , in PEMF-treated HUVECs. Western blotting was used to assess the protein expression levels of Akt, mTOR and TGF- $\beta$ in PEMF- and HTY-treated HUVECs. PEMF treatment alone had no effect on the protein expression levels of Akt; however, combined treatment with HTY significantly upregulated these levels (Fig. 5A). PEMF treatment alone upregulated the protein expression levels of mTOR, and this effect was further enhanced by combined treatment with HTY (Fig. 5B). TGF- $\beta$ protein expression levels demonstrated the same pattern of effect as Akt (Fig. 5C). Both HTY and PEMF had no effect on the protein expression level of p53.

\section{Discussion}

In the present study, several novel findings were demonstrated. To the best of our knowledge, we demonstrated for the first time that HTY promotes HUVEC proliferation mediated by PEMFs in a time-dependent manner. Secondly, the data from the study revealed that HTY and PEMFs serve synergistic roles in anti-apoptosis and ameliorating mitochondrial dysfunction induced by $\mathrm{H}_{2} \mathrm{O}_{2}$ incubation. Thirdly, to clarify the anti-apoptosis and pro-proliferation effects of HTY following PEMF treatment, the mRNA and protein expression levels of Akt, mTOR, TGF- $\beta$ and p53 were detected. It was demonstrated that HTY increases the transcription activities of Akt, mTOR and TGF- $\beta$, but not p53, in HUVECs in vitro. To the best of our knowledge, the present study was the first to reveal the molecular mechanism of co-treatment with PEMFs and HTY on the biological characteristics in HUVECs. These results not only facilitate understanding of the mechanisms underlying the effects of HTY on HUVECs, but also improve the view of co-treatment of HTY and PEMFs that may serve as potential therapeutic strategy in wound healing in the future.

Previous studies have demonstrated that PEMFs are a non-invasive and non-pharmacological intervention, and have been reported to repair damaged tissue (16-18). Several clinical studies have demonstrated that PEMFs can effectively alleviate tissue swell, traumatic pain and promote wound healing $(19,20)$. Guerriero et al (21) described the first reported case of an innovative PEMF therapy, emysimmetric bilateral stimulation (EBS), used to successfully treat refractory skin ulcers in two elderly and fragile patients. They demonstrated a supportive role of PEMFs in the treatment of skin ulceration in diabetes, which was suggestive of a potential benefit of EBS for this clinical condition. HTY is a major phenolic compound in virgin olive oil in both free and complex forms. Catalán et al (22) revealed the protective effect of HTY and its predominant plasmatic human metabolites, synthetized for the first time by using an intestinal cell model, which may be responsible in part for the protection against endothelial dysfunction. However, no previous findings have suggested that the combined treatment of PEMFs with HTY is a potentially promising therapeutic strategy for treating wound healing. To further investigate the mechanism of PEMF- and HTY-mediated neovascularization, the present study investigated the effects of co-treatment of PEMF and HTY on endothelial biological modification in vitro. The synergistic effect of PEMFs and HTY were identified, which may improve multiple endothelial functions including migration, proliferation, anti-apoptosis and mitochondrial dysfunction, suggesting the therapeutic potential of PEMF- and HTY-combined treatment on pre-existing endothelial vasculature. However, other mechanisms involved in PEMF- and HTY-mediated neovascularization may exist; therefore, several limitations of the current research should be highlighted. For example, the present study used cell models, and the findings may not be extrapolated directly to animal models and humans. The gene activation and protein production of the HTY-stimulated HUVECs will require further study to confirm the beneficial effects of PEMFs on wound healing in vivo. Precaution should be taken when applying the results of this study to patients. Nevertheless, these results provide a basis for future studies to investigate whether the effects of co-treatment of PEMFs and HTY are also applicable in a clinical setting.

In conclusion, the results of the present study revealed that combined exposure of PEMFs and HTY to HUVECs demonstrated protective effects on proliferation, migration and apoptosis in vitro, implicating the potential benefits of this treatment for wound healing in the future.

\section{References}

1. Janis JE, Attinger CE and Lavery L: Introduction to 'current concepts in wound healing: Update 2016'. Plast Reconstr Surg 138 (3 Suppl): 7S-8S, 2016.

2. Yoshida S, Yoshimoto H, Hirano A and Akita S: Wound healing and angiogenesis through combined use of a vascularized tissue flap and adipose-derived stem cells in a rat hindlimb irradiated ischemia model. Plast Reconstr Surg 137: 1486-1497, 2016.

3. Athanasiou A, Karkambounas S, Batistatou A, Lykoudis E, Katsaraki A, Kartsiouni T, Papalois A and Evangelou A: The effect of pulsed electromagnetic fields on secondary skin wound healing: Aexperimental study. Bioelectromagnetics 28: 362-368, 2007.

4. Delle Monache S, Alessandro R, Iorio R, Gualtieri G and Colonna R: Extremely low frequency electromagnetic fields (ELF-EMFs) induce in vitro angiogenesis process in human endothelial cells. Bioelectromagnetics 29: 640-648, 2008.

5. Li F, Lei T, Xie K, Wu X, Tang C, Jiang M, Liu J, Luo E and Shen G: Effects of extremely low frequency pulsed magnetic fields on diabetic nephropathy in streptozotocin-treated rats. Biomed Eng Online 15: 8, 2016.

6. Martino CF, Perea H, Hopfner U, Ferguson VL and Wintermantel E: Effects of weak static magnetic fields on endothelial cells. Bioelectromagnetics 31: 296-301, 2010. 
7. Li RL, Huang JJ, Shi YQ, Hu A, Lu ZY, Weng L, Wang SQ, Han YP, Zhang L, Hao CN and Duan JL: Pulsed electromagnetic field improves postnatal neovascularization in response to hindlimb ischemia. Am J Transl Res 7: 430-444, 2015.

8. Cheing GL, Li X, Huang L, Kwan RL and Cheung KK: Pulsed electromagnetic fields (PEMF) promote early wound healing and myofibroblast proliferation in diabetic rats. Bioelectromagnetics 35: 161-169, 2014.

9. Goudarzi I, Hajizadeh S, Salmani ME and Abrari K: Pulsed electromagnetic fields accelerate wound healing in the skin of diabetic rats. Bioelectromagnetics 31: 318-323, 2010.

10. Lamy S, Ben Saad A, Zgheib A and Annabi B: Olive oil compounds inhibit the paracrine regulation of TNF-alpha-induced endothelial cell migration through reduced glioblastoma cell cyclooxygenase-2 expression. J Nutr Biochem 27: 136-145, 2016.

11. Morbidelli L: Polyphenol-based nutraceuticals for the control of angiogenesis: Analysis of the critical issues for human use. Pharmacol Res 111: 384-393, 2016.

12. Zrelli H, Kusunoki M and Miyazaki H: Role of hydroxytyrosol-dependent regulation of $\mathrm{HO}-1$ expression in promoting wound healing of vascular endothelial cells via Nrf2 De Novo synthesis and stabilization. Phytother Res 29: 1011-1018, 2015.

13. Scoditti E, Calabriso N, Massaro M, Pellegrino M, Storelli C, Martines G, De Caterina R and Carluccio MA: Mediterranean diet polyphenols reduce inflammatory angiogenesis through MMP-9 and COX-2 inhibition in human vascular endothelial cells: A potentially protective mechanism in atherosclerotic vascular disease and cancer. Arch Biochem Biophys 527: 81-89, 2012.

14. Ferroni L, Bellin G, Emer V, Rizzuto R, Isola M, Gardin C and Zavan B: Treatment by Therapeutic Magnetic Resonance (TMR) increases fibroblastic activity and keratinocyte differentiation in an in vitro model of 3D artificial skin. J Tissue Eng Regenerat med 11: 1332-1342, 2015.

15. Livak KJ and Schmittgen TD: Analysis of relative gene expression data using real-time quantitative PCR and the 2(-Delta Delta C(T)) method. Methods 25: 402-408, 2001.
16. Goto T, Fujioka M, Ishida M, Kuribayashi M, Ueshima K and Kubo T: Noninvasive up-regulation of angiopoietin-2 and fibroblast growth factor-2 in bone marrow by pulsed electromagnetic field therapy. J Orthop Sci 15: 661-665, 2010.

17. Osti L, Buono AD and Maffulli N: Pulsed electromagnetic fields after rotator cuff repair: A randomized, controlled study. Orthopedics 38: e223-e228, 2015.

18. Kim YT, Hei WH, Kim S, Seo YK, Kim SM, Jahng JW and Lee JH: Co-treatment effect of pulsed electromagnetic field (PEMF) with human dental pulp stromal cells and FK506 on the regeneration of crush injured rat sciatic nerve. Int J Neurosci 125 774-783, 2015.

19. Bagnato GL, Miceli G, Marino N, Sciortino D and Bagnato GF: Pulsed electromagnetic fields in knee osteoarthritis: A double blind, placebo-controlled, randomized clinical trial. Rheumatology (Oxford) 55: 755-762, 2016.

20. Menini M, Bevilacqua M, Setti P, Tealdo T, Pesce P and Pera P: Effects of pulsed electromagnetic fields on swelling and pain after implant surgery: A double-blind, randomized study. Int J Oral Maxillofac Surg 45: 346-353, 2016.

21. Guerriero F, Botarelli E, Mele G, Polo L, Zoncu D, Renati P, Sgarlata C, Rollone M, Ricevuti G, Maurizi N, et al: Effectiveness of an innovative pulsed electromagnetic fields stimulation in healing of untreatable skin ulcers in the frail elderly: Two case reports. Case Rep Dermatol Med 2015: 576580, 2015.

22. Catalán Ú, López de Las Hazas MC, Rubió L, Fernández-Castillejo S, Pedret A, de la Torre R, Motilva MJ and Solà R: Protective effect of hydroxytyrosol and its predominant plasmatic human metabolites against endothelial dysfunction in human aortic endothelial cells. Mol Nutr Food Res 59: 2523-2536, 2015. 